\title{
Modulating Regional Motor Cortical Excitability with Noninvasive Brain Stimulation Results in Neurochemical Changes in Bilateral Motor Cortices
}

\author{
๑DVelicia Bachtiar, ${ }^{1 *}$ (DAinslie Johnstone, ${ }^{1,2 *}{ }^{\circledR}$ Adam Berrington, ${ }^{1}$ Clark Lemke, ${ }^{1}$-Heidi Johansen-Berg, ${ }^{1}$ \\ ㄴzay Emir, ${ }^{1}$ and ${ }^{\circledR C}$ Charlotte J. Stagg ${ }^{1,2}$ \\ ${ }^{1}$ Nuffield Department of Clinical Neurosciences, Functional Magnetic Resonance Imaging of the Brain (FMRIB), Wellcome Centre for Integrative \\ Neuroimaging, University of Oxford, Oxford, OX3 9DU, United Kingdom, and 2Department of Psychiatry, Oxford Centre for Human Brain Activity, \\ Wellcome Centre for Integrative Neuroimaging, University of Oxford, Oxford, OX3 7JX, United Kingdom
}

Learning a novel motor skill is dependent both on regional changes within the primary motor cortex (M1) contralateral to the active hand and also on modulation between and within anatomically distant but functionally connected brain regions. Interregional changes are particularly important in functional recovery after stroke, when critical plastic changes underpinning behavioral improvements are observed in both ipsilesional and contralesional M1s. It is increasingly understood that reduction in GABA in the contralateral M1 is necessary to allow learning of a motor task. However, the physiological mechanisms underpinning plasticity within other brain regions, most importantly the ipsilateral M1, are not well understood. Here, we used concurrent two-voxel magnetic resonance spectroscopy to simultaneously quantify changes in neurochemicals within left and right M1s in healthy humans of both sexes in response to transcranial direct current stimulation (tDCS) applied to left M1. We demonstrated a decrease in GABA in both the stimulated (left) and nonstimulated (right) M1 after anodal tDCS, whereas a decrease in GABA was only observed in nonstimulated M1 after cathodal stimulation. This GABA decrease in the nonstimulated M1 during cathodal tDCS was negatively correlated with microstructure of M1:M1 callosal fibers, as quantified by diffusion MRI, suggesting that structural features of these fibers may mediate GABA decrease in the unstimulated region. We found no significant changes in glutamate. Together, these findings shed light on the interactions between the two major network nodes underpinning motor plasticity, offering a potential framework from which to optimize future interventions to improve motor function after stroke.

Key words: DTI; GABA; M1; MRS; plasticity; tDCS

\section{Significance Statement}

Learning of new motor skills depends on modulation both within and between brain regions. Here, we use a novel two-voxel magnetic resonance spectroscopy approach to quantify GABA and glutamate changes concurrently within the left and right primary motor cortex (M1) during three commonly used transcranial direct current stimulation montages: anodal, cathodal, and bilateral. We also examined how the neurochemical changes in the unstimulated hemisphere were related to white matter microstructure between the two M1s. Our results provide insights into the neurochemical changes underlying motor plasticity and may therefore assist in the development of further adjunct therapies.

\section{Introduction}

It has been shown consistently that plasticity in the motor system, whether underlying the learning of novel motor skills or their

\footnotetext{
Received Nov. 12, 2017; revised May 9, 2018; accepted May 15, 2018.

Author contributions: V.B., A.B., C.L., H.J.-B., U.E., and C.J.S. designed research; V.B., A.B., C.L., and U.E. performed research; V.B. and A.J. analyzed data; A.J. and C.J.S. wrote the paper.

V.B. was funded by a Clarendon Fund scholarship. A.J. is funded by a Medical Research Council studentship. C.J.S. holds a Sir Henry Dale Fellowship funded by the Wellcome Trust and the Royal Society (Grant 102584/Z/13/Z). H.J.B. holds a Wellcome Principal Research Fellowship (Grant 110027/Z/15/Z). The Wellcome Centre for Integrative Neuroimaging is supported by core funding from the Wellcome Trust (Grant 203139/Z/16/Z).

The authors declare no competing financial interests.

*V.B. and A.J. contributed equally to this work.
}

relearning after stroke, involves changes within and between a network of anatomically distributed motor regions. In particular, it is clear from studies examining the neural control of unilateral

U. Emir's present address: Purdue University School of Health Sciences, West Lafayette, Indiana 47907.

A. Berrington's present address: Division of Neuroradiology, Johns Hopkins Medicine, Baltimore, Maryland MD 21205.

Correspondence should be addressed to Dr. Charlotte J. Stagg, FMRIB, John Radcliffe Hospital, Headington, Oxford 0X3 9DU, UK. E-mail: charlotte.stagg@ndcn.ox.ac.uk.

DOI:10.1523/JNEUROSCI.2853-17.2018

Copyright $\odot 2018$ Bachtiar, Johnstone et al.

This is an open-access article distributed under the terms of the Creative Commons Attribution License Creative Commons Attribution 4.0 International, which permits unrestricted use, distribution and reproduction in any medium provided that the original work is properly attributed. 
motor behaviors that the interaction between the two primary motor cortices (M1s) is vital for motor learning (Perez and Cohen, 2009; Reis et al., 2009; Di Pino et al., 2014). However, little is known about exactly how this interaction occurs and what physiological changes in distant nodes underpin interregional plasticity. Animal and computational models have suggested that network-level plasticity may depend on the relationship between high-frequency oscillations within the network-nodes, which are driven at least in part by regional GABAergic activity (Cabral et al., 2011; Hall et al., 2011; Nowak et al., 2017). It is plausible to hypothesize, therefore, that decreases in GABA in distant network nodes are necessary to increase coherence, change connectivity, and therefore allow network-level plasticity to occur. Here, we tested this hypothesis by studying neurochemical changes underpinning plasticity in both M1s simultaneously using transcranial direct current stimulation (tDCS).

Anodal tDCS to M1 is thought to induce long-term potentiation (LTP)-like changes within the stimulated region (Hess et al., 1996; Fritsch et al., 2010; Stagg and Nitsche, 2011; Monte-Silva et al., 2013) and has been demonstrated to affect regional neurochemistry in a manner similar to motor learning, most notably resulting in a regional decrease in GABA concentration (FloyerLea et al., 2006; Stagg et al., 2009, 2011a; Stagg and Nitsche, 2011; Bachtiar et al., 2015).

The neurochemical changes due to tDCS are reflected in behavioral changes, with anodal tDCS improving learning if applied during practice of a motor task (Nitsche et al., 2003; Galea et al., 2011; Stagg et al., 2011a; Cuypers et al., 2013; Kim et al., 2014; Sriraman et al., 2014; Amadi et al., 2015; Waters et al., 2017). This beneficial effect of anodal tDCS on motor learning has led to tDCS being suggested as a putative adjunct therapy to enhance motor rehabilitation after stroke, with some success in small proof-of-principle studies (Fregni et al., 2005; Hummel and Cohen, 2005; Hummel et al., 2005; Kim et al., 2006; Boggio et al., 2007; Lindenberg et al., 2010; Allman et al., 2016).

In addition to applying anodal tDCS to the ipsilesional hemisphere after stroke, some studies have shown that applying cathodal tDCS to the contralesional hemisphere to reduce local activity here also leads to behavioral improvements in the paretic hand. This functional improvement is accompanied by increased activity in the ipsilesional M1, consistent with the hypothesis of interhemispheric imbalance in this patient group (Fregni et al., 2005; Boggio et al., 2007; Kim et al., 2010; Stagg et al., 2012; O'Shea et al., 2014). However, the mechanism by which contralesional tDCS can increase activity in the contralateral and ipsilesional M1 is not understood.

In this study, we used a novel two-voxel magnetic resonance spectroscopy (MRS) approach to quantify GABA and glutamate changes concurrently within the left and right M1 during the three most commonly used tDCS montages: anodal to left M1, cathodal to left M1, and bilateral, in which electrodes are placed over both M1s. We also examined how the neurochemical changes in the unstimulated hemisphere were related to white matter microstructure between the two M1s.

\section{Materials and Methods \\ Participants}

Twelve healthy participants (two male, aged 20-32 years, mean 25 years) gave their informed consent to participate in this study in accordance with ethical approval from the East London Research Ethics Committee (reference \#10/H0703/50). All were right-handed as assessed by the Edinburgh Handedness Inventory (Oldfield, 1971).

\section{Experimental design}

Participants undertook five experimental sessions: four of which were MRS scans at $7 \mathrm{~T}$ to assess changes in GABA and glutamate concentrations in both M1s simultaneously due to sham, anodal, or cathodal tDCS applied to the left M1 or to bilateral tDCS with the anode positioned over left M1 (see Fig. 1 for an experimental outline).

To relate the magnitude of any changes in neurochemicals across anatomically distant regions, we additionally acquired diffusion tensor imaging (DTI) measures of white matter microstructure in a fifth imaging session performed at $3 \mathrm{~T}$.

All MRS-tDCS sessions were separated by at least 1 week and the order of the sessions was counterbalanced across the group. Two subjects did not complete the bilateral MRS-tDCS session due to personal time constraints. In each MRS-tDCS session, MRS spectra were acquired over five time points: at baseline, during tDCS, and at three time points after tDCS: Post $1=\sim 1-13$ min after tDCS, Post $2=\sim 14-26$ min after tDCS, and Post $3=\sim 27-39$ min after tDCS (see Figure 1 for experimental outline). Subjects watched a nature documentary for the duration of the experiment.

\section{$t D C S$}

A DC stimulator (Magstim) delivered a $1 \mathrm{~mA}$ current to the brain via electrodes measuring $5 \times 7 \mathrm{~cm}$ (Easycap). For the anodal, cathodal, and sham sessions, one electrode was centered over the left M1 positioned $5 \mathrm{~cm}$ lateral to $\mathrm{Cz}$ and the other over the contralateral supraorbital ridge. For the bilateral session, the anode was placed over the left M1 and the cathode over the right $\mathrm{M}$ 1, each electrode positioned $5 \mathrm{~cm}$ lateral to $\mathrm{Cz}$. Although this method of electrode placement does not take into account individual's head sizes, we believe that differences in electrode placement between individuals are likely to be relatively minor.

High-chloride electrolyte gel (Easycap) was used as the conducting medium between the scalp and electrodes. The electrodes contained $5 \mathrm{k} \Omega$ resistors and extension leads connected the stimulator, which was located outside of the magnetic field, to the subject positioned in the scanner. For the real stimulation conditions (anodal, cathodal, bilateral) the current was ramped up over $10 \mathrm{~s}$ and was then held at $1 \mathrm{~mA}$ for $10 \mathrm{~min}$ before being ramped down over $10 \mathrm{~s}$. For sham stimulation, the DC stimulator was ramped up for $10 \mathrm{~s}$ and then switched off, as described previously (Stagg et al., 2011b).

\section{MRS data acquisition}

For full details of the MRS acquisition approach, see Lemke et al. (2015). Briefly, MRS data were acquired on a 7 T Siemens MR system with a 32-channel receive array head-coil. $\mathrm{T}_{1}$-weighted images (MPRAGE, $192 \times 1 \mathrm{~mm}$ axial slices, TR/TE $=2200 / 2.82 \mathrm{~ms}$, flip-angle $=7 \circ, \mathrm{FOV}$ $192 \times 100$ ) were used to place a $2 \times 2 \times 2 \mathrm{~cm}$ voxel of interest over the left and right precentral knobs, a known landmark for hand motor representation (Yousry et al., 1997).

Spectra were measured using the sLASER sequence $(\mathrm{TR} / \mathrm{TE}=7000 / 30$ ms) (Oz and Tkáč, 2011) with VAPOR water suppression (Tkáč et al., 1999). Spectra were acquired in an interleaved fashion from the two voxels (64 transients each) (Lemke et al., 2015). Each block of MRS data acquisition took $\sim 12 \mathrm{~min}$.

\section{MRI acquisition}

MRI data were acquired using a 3 T Siemens Verio MRI System and a 32-channel receive head coil. A whole-brain high-resolution structural image ( $1.0 \mathrm{~mm}$ isotropic) was also collected using a multigradient echo sequence to enable anatomic registration $(\mathrm{TE} 1=1.79 \mathrm{~ms}$, TE2 $=3.65$ $\mathrm{ms}, \mathrm{TE} 3=5.51 \mathrm{~ms}, \mathrm{TE} 4=7.37 \mathrm{~ms}, \mathrm{TR}=2530 \mathrm{~ms}, \mathrm{FOV}=256 \times 256)$.

To relate the microstructure of the corpus callosum (CC) to the change in MRS metrics acquired at $7 \mathrm{~T}$, echoplanar diffusion weighted imaging was acquired (diffusion directions $=64$, b-value $=1500 \mathrm{~s} / \mathrm{mm}^{2}$, voxel dimensions $=2 \times 2 \times 2 \mathrm{~mm}, 64$ slices, $\mathrm{TR}=8900 \mathrm{~ms}, \mathrm{TE}=91 \mathrm{~ms})$.

We also acquired fMRI scans to enable functional localization of M1 in each subject. A total of 130 echoplanar volumes were collected $(3 \mathrm{~mm}$ isotropic voxels, $\mathrm{TE}=30 \mathrm{~ms}, \mathrm{TR}=3000 \mathrm{~ms}, \mathrm{FOV}=192 \times 192 \mathrm{~mm}$ ) while the participant performed visually cued hand tapping at $1 \mathrm{~Hz}$ with either the left or right hand, interspersed with blocks of rest. Blocks of movement and rest were $30 \mathrm{~s}$ long. 


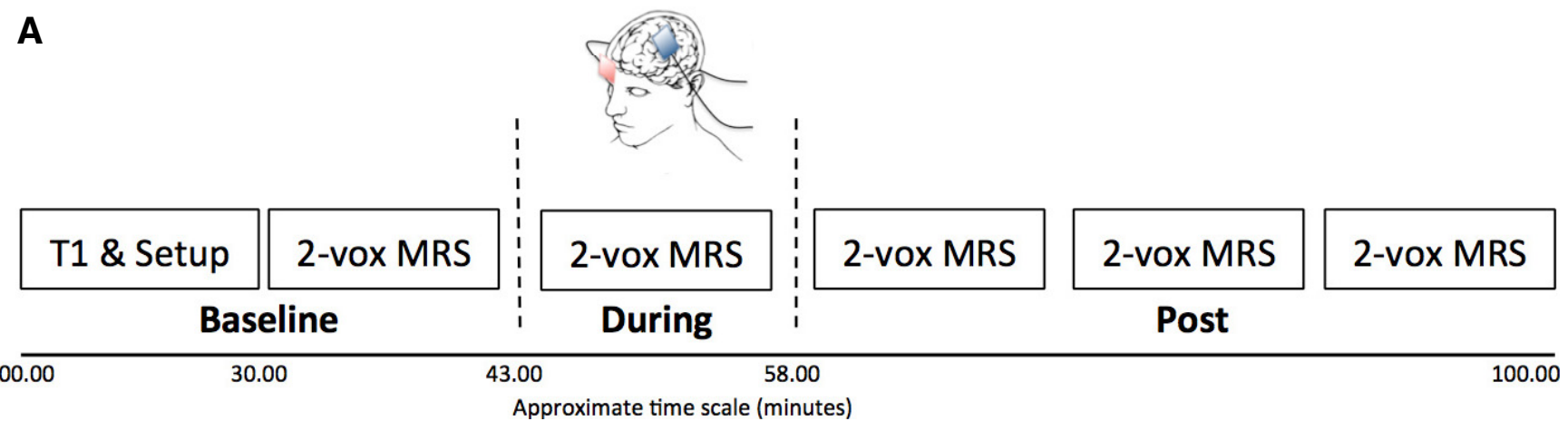

B

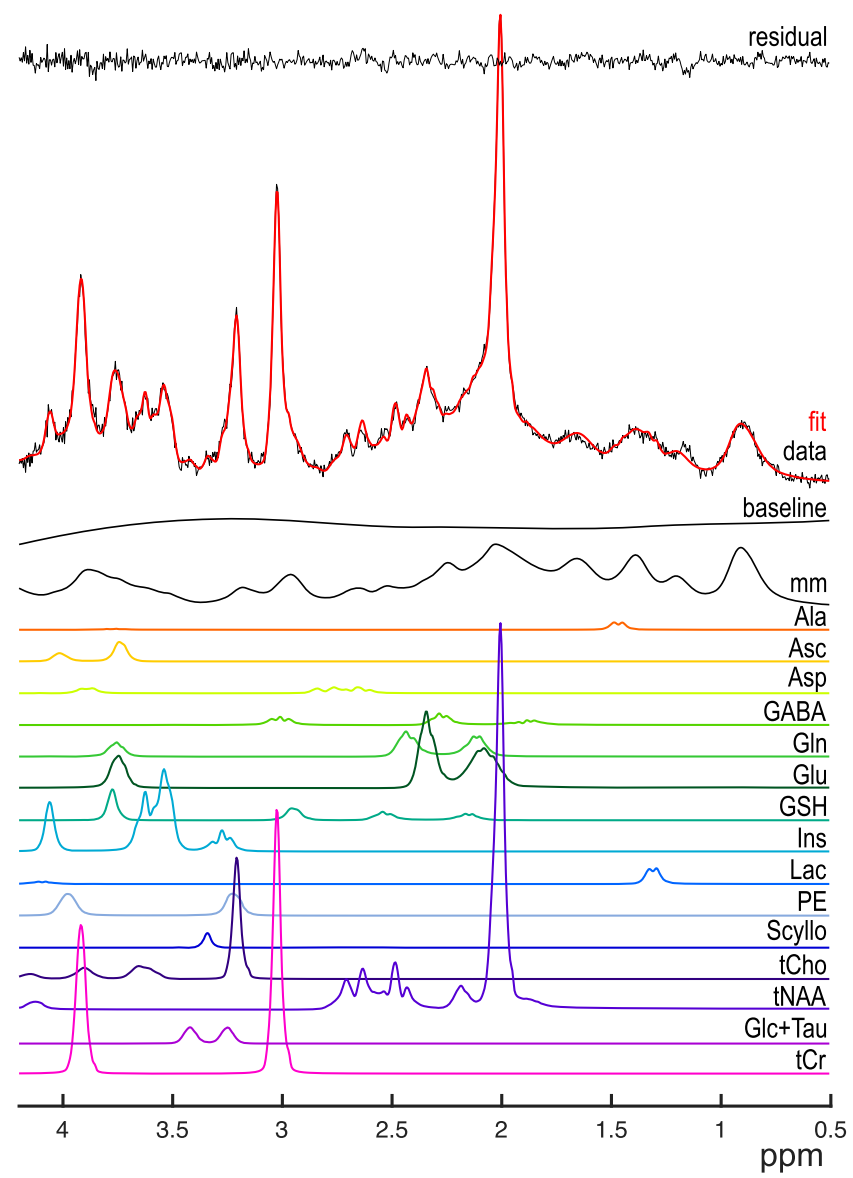

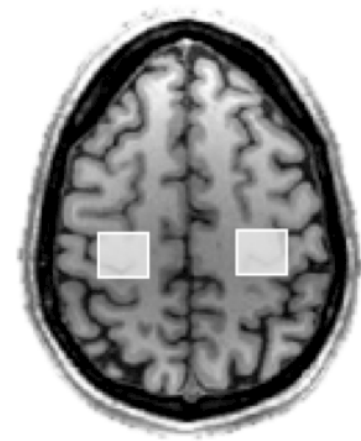

$z=196$

Figure 1. A, Schematic outlining the protocol of the 7 T MRS sessions. $B$, Representative spectrum from one acquisition block from one voxel, as analyzed by LCModel, showing the raw data (red) as well as the individual metabolite fits (colored lines) and the residual noise (black). Inset shows the location of both voxels from which MRS was acquired.

\section{Data analysis}

MRS analysis

As has been described previously (Lemke et al., 2015), standard preprocessing, including eddy current correction and zero-order phasing of array coil spectra, was performed using in-house scripts. Any residual water signal was removed from the metabolite spectra using HankelLanczos singular value decomposition (Cabanes et al., 2001). Neurochemicals were quantified using LCModel analysis (RRID:SCR_014455) (Provencher, 2001). The following criteria were applied for excluding spectra with poor quality: (1) Cramer-Rao lower bounds $>50 \%$, (2) water linewidths at full width at half-maximum (FWHM) $>15 \mathrm{~Hz}$, and (3) signal-to-noise ratio $<10$. Of the 460 spectral blocks acquired (five time points from two voxels in 12 subjects with three to four sessions per subject), 15 blocks from five subjects were excluded on these criteria for the GABA measurements and two spectra from one subject were excluded for the glutamate measurement.

As is common, neurochemical concentrations are presented here as ratios relative to total creatine (tCr) (Soares and Law, 2009; Mullins et al., 2014). To examine the relationship between individuals' baseline GABA levels and MRI measures, the mean baseline GABA:Cr value for right and left M1 in each subject across the sessions was calculated.

\section{fMRI analysis}

Analysis was performed using FEAT (FMRI Expert Analysis Tool) version 6.00, part of FSL (FMRIB's Software Library, www.fmrib.ox.ac.uk/fsl, RRID:SCR_002823) (Smith et al., 2004; Woolrich et al., 2009; Jenkinson et al., 2012). Registration to high-resolution structural T1-weighted images was performed using FLIRT. Registration from structural images to 
standard space was then further refined using FNIRT nonlinear registration (Andersson et al., 2007a,b).

The following prestatistics processing was then applied: motion correction using MCFLIRT (Jenkinson et al., 2002), nonbrain removal using BET (Smith, 2002; Brain Extraction Tool, RRID:SCR_014586), spatial smoothing using a Gaussian kernel of FWHM $5 \mathrm{~mm}$, grand-mean intensity normalization for the entire $4 \mathrm{D}$ dataset by a single multiplicative factor, and high-pass temporal filtering (Gaussian-weighted least-squares straight line fitting with $\sigma=57.0$ s). Independent component analysis-based exploratory analysis was performed using MELODIC (Beckmann et al., 2005) to investigate the possible presence of unexpected artifacts or activation. FSL Motion Outliers was run on all functional scans to identify any time points corrupted by large motion. The resulting confound matrix was then included in the general linear model to remove the effects of these time points on the resulting analyses.

For each participant, contrasts of left press $>$ rest and right press $>$ rest were performed with a $Z$ threshold of 2.3 and cluster $P$ threshold of 0.05 . Group-level analysis was then performed to find group average clusters of activation $(Z$ threshold $=2.3$, cluster $P$ threshold $=0.05)$. These group-level clusters were then transformed into individuals' DTI space using FLIRT (Andersson et al., 2007a,b) and were used as functionally defined M1 masks.

\section{DTI analysis}

Diffusion data were processed using tools from FSL (Smith et al., 2004; Jenkinson et al., 2012). Diffusion-weighted scans were first corrected for eddy-current distortions using eddycorrect and then brain extracted using BET. Local diffusion tensors were then fit using the dtifit command in FSL. Markov Chain Monte Carlo sampling was then used to build the diffusion orientation distribution functions for each voxel (Behrens et al., 2007) using Bedpostx (BEDPOSTX; FMRIB; Behrens et al., 2003, 2007), modeling a total of up to two fibers per voxel and using total of 1000 iterations.

Tractography. Tracts running from the CC to the motor cortices were estimated using PROBTRACKX2 (Behrens et al., 2003, 2007). A mask of the CC was created using the Juelich thr 25 atlas and transformed into each participant's DTI space. Bilateral M1s were functionally defined during the same $3 \mathrm{~T}$ scan. The CC mask was used as the seed mask, with functionally defined bilateral M1s as a one-way waypoint, meaning that only pathways that passed through the CC and reached at least M1 would be retained. A total of 5000 individual pathways were generated from each voxel within the seed mask using a step length of $0.5 \mathrm{~mm}$ and a maximum of 2000 steps. A cosine curvature threshold of $0.2\left(\sim 80^{\circ}\right)$ was used to limit how sharply pathways could deflect during tract generation. This procedure generated a tract map for each participant in which voxel values represented the total number of pathways passing through each brain voxel.

For each participant, this tract map was thresholded at $>7500$, binarized, and then the overlap between this mask and the CC mask was used to define a M1-M1 CC mask. The mean fractional anisotropy (FA) value across all voxels within this mask was then extracted and used in subsequent correlation analyses.

A first control region, the area of the CC carrying tracts connecting left M1 to right somatosensory cortex was defined using the method described above, but using Harvard-Oxford defined right somatosensory cortex and functional defined left M1 as waypoints in PROBTRACKX2. This tract was thresholded to $<25$ and masked with the Harvard-Oxford CC mask. A second control region in right corticospinal tract (rCST) was defined by using the right $\mathrm{M} 1$ as a seed point and right internal capsule as a waypoint, with the same parameters as for the M1-M1 tract. For each participant, this tract was thresholded at $>2000$, binarized, and then the region of interest was defined as the area within this tract mask falling within a $25 \times 25 \times 25$ voxel volume around the center of the internal capsule. This approach was taken to ensure that the volume of the rCST mask and the M1-M1 CC mask were not significantly different (paired $t$ test, $t_{(11)}=1.749, p=0.11$ ).

\section{Results}

We first wanted to test whether there were any baseline differences in neurochemicals across sessions. We therefore performed a repeatedmeasures ANOVA (RM-ANOVA) with within-subject factors of stimulation condition (anodal, cathodal, bilateral, sham), hemisphere (right M1, left M1), and neurochemical (GABA, Glu). This demonstrated no significant main effect of stimulation condition $\left(F_{(3,21)}=0.928, p=0.445\right)$, no stimulation by hemisphere interaction $\left(F_{(3,21)}=2.357, p=0.101\right)$, and no significant stimulation by neurochemical interaction $\left(F_{(3,21)}=2.515, p=0.086\right)$.

Therefore, to control for intersubject differences in baseline neurochemical concentration, we calculated the change in each neurochemical from baseline at each time point for all subjects and sessions and used these values in all subsequent analyses.

All neurochemical concentrations presented here are referenced to $\mathrm{tCr}(\mathrm{Cr}+\mathrm{PCr}=\mathrm{tCr})$. We therefore wished to test for any changes in creatine over time that might potentially affect our results. We found no change in tCr over time in either M1 (RMANOVA for each M1 separately with one factor of stimulation condition (anodal, cathodal, bilateral, sham) and one factor of time (baseline, during, Post 1, Post 2, Post 3); no main effect of stimulation or time, or stimulation by time interaction for either M1 (all $p>0.05)$.

\section{tDCS to the left M1 leads to significant changes in neurochemicals in both hemispheres}

We wished to investigate whether there were any significant changes in neurochemicals in response to tDCS. A RM-ANOVA with within-subjects factors of stimulation condition (anodal, cathodal, bilateral, sham), hemisphere (right M1, left M1), neurochemical (glutamate, GABA), and time (during, Post 1, Post 2, Post 3) demonstrated a significant main effect of stimulation condition $\left(F_{(3,15)}=5.235, p=0.011\right)$; no significant main effect of hemisphere $\left(F_{(1,5)}=0.006, p=0.940\right)$; no main effect of neurochemical $\left(F_{(1,5)}=0.890, p=0.890\right)$; and no significant main effect of time $\left(F_{(3,15)}=0.904, p=0.463\right)$. Importantly, we demonstrated a significant interaction between stimulation condition and neurochemical $\left(F_{(3,15)}=4.223, p=0.024\right)$ (see Table 1 for full results).

To explore the effects of the different stimulation conditions further, we investigated whether there were significant changes in the left and right M1s due to tDCS when the neurochemicals and hemispheres were considered separately using RM-ANOVAs and the within-subjects factors of stimulation condition (anodal, cathodal, bilateral, sham) and time (during, Post 1, Post 2, Post 3 ). We found a significant main effect of stimulation condition on the change in GABA concentration within both left and right M1s (left M1, GABA: $F_{(3,24)}=4.739, p=0.016$; right M1, GABA: $\left.F_{(3,24)}=7.513, p=0.001\right)$. There was no significant main effect of time (left M1, GABA: $F_{(3,15)}=2.755, p=0.079$; right M1, GABA: $\left.F_{(3,24)}=0.030, p=0.993\right)$ and no interaction between stimulation and time (left M1, GABA: $F_{(9,45)}$; right M1, GABA: $F_{(9,72)}=$ $1.311, p=0.246)$ in either hemisphere.

However, tDCS had no significant effect of stimulation on glutamate concentration within either hemisphere (left M1, glutamate: main effect of stimulation condition: $F_{(3,27)}=2.110, p=$ 0.122; main effect of time: $F_{(3,27)}=0.535, p=0.669$; stimulation condition by time interaction: $F_{(9,81)}=0.597, p=0.796$; left M1, glutamate: main effect of stimulation condition: $F_{(3,27)}=1.329$, $p=0.118$; main effect of time: $F_{(3,27)}=1.236, p=0.316$; stimulation condition by time interaction: $\left.F_{(9,81)}=1.131, p=0.351\right)$. 
Table 1. Full results of RM-ANOVA with one factor of stimulation condition (anodal, cathodal, bilateral, sham), one factor of hemisphere (right M1, left M1), one factor of neurochemical (glutamate, GABA), and one factor of time (during, Post 1, Post 2, Post 3)

\begin{tabular}{|c|c|c|c|c|}
\hline Effect & $\mathrm{df}$ & $F$ & $p$ & $\eta^{2}$ \\
\hline Stimulation condition & 3 & 5.235 & 0.011 & 0.511 \\
\hline Error (stimulation condition) & 15 & & & \\
\hline Hemisphere & 1 & 0.006 & 0.940 & 0.001 \\
\hline Error (hemisphere) & 5 & & & \\
\hline neurochemical & 1 & 0.890 & 0.389 & 0.151 \\
\hline Error (neurochemical) & 5 & & & \\
\hline Time & 3 & 0.904 & 0.462 & 0.153 \\
\hline Error (time) & 15 & & & \\
\hline Stimulation condition*hemisphere & 3 & 0.538 & 0.663 & 0.097 \\
\hline Error (stimulation condition * hemisphere) & 15 & & & \\
\hline Stimulation condition * neurochemical & 3 & 2.101 & 0.024 & 0.458 \\
\hline Error (stimulation condition * neurochemical) & 15 & & & \\
\hline Hemisphere* neurochemical & 1 & 0.002 & 0.969 & $<0.001$ \\
\hline Error (hemisphere * neurochemical) & 5 & & & \\
\hline Stimulation condition * time & 9 & 1.648 & 0.130 & 0.248 \\
\hline Error (stimulation condition * time) & 45 & & & \\
\hline Hemisphere* time & 3 & 1.120 & 0.372 & 0.183 \\
\hline Error (hemisphere * time) & 15 & & & \\
\hline Time* neurochemical & 3 & 0.047 & 0.618 & 0.109 \\
\hline Error (time * neurochemical) & 15 & & & \\
\hline Stimulation condition *hemisphere* neurochemical & 3 & 0.550 & 0.407 & 0.171 \\
\hline Error (stimulation condition * hemisphere *neurochemical) & 15 & & & \\
\hline Stimulation condition * hemisphere ${ }^{*}$ time & 9 & 0.306 & 0.969 & 0.058 \\
\hline Error (stimulation condition * hemisphere * time) & 45 & & & \\
\hline Hemisphere ${ }^{*}$ neurochemical ${ }^{*}$ time & 3 & 0.787 & 0.520 & 0.136 \\
\hline Error (stimulation condition * neurochemical * time) & 15 & & & \\
\hline Stimulation condition * neurochemical * time & 9 & 0.885 & 0.545 & 0.150 \\
\hline Error (stimulation condition * neurochemical * time) & 45 & & & \\
\hline Stimulation condition * neurochemical ${ }^{*}$ hemisphere $*$ time & 9 & 1.671 & 0.125 & 0.250 \\
\hline Error (stimulation condition neurochemical * hemisphere * time) & 45 & & & \\
\hline
\end{tabular}

Both anodal and cathodal tDCS to left M1 lead to a significant decrease in GABA in the unstimulated right $\mathrm{M1}$

To investigate the changes in GABA in each hemisphere due to each stimulation protocol directly, we then tested each real stimulation condition against the sham session using RM-ANOVAs with one factor of stimulation condition (real, sham) and one factor of time (during, Post 1, Post 2, Post 3). This approach demonstrated a decrease in GABA concentration in response to anodal tDCS compared with sham in both the stimulated left M1 (main effect of stimulation condition: $F_{(1,8)}=8.964, p=0.017$, no significant main effect of time: $F_{(3,24)}=2.711, p=0.067$; no stimulation by time interaction: $\left.F_{(3,24)}=0.611, p=0.614\right)$ and the nonstimulated right M1 (main effect of stimulation condition: $F_{(1,11)}=7.898, p=0.017$; no significant effect of time: $F_{(3,33)}=1.278, p=0.298$; no stimulation by time interaction: $F_{(3,33)}=0.816, p=0.494$; Fig. $\left.2 A\right)$.

Cathodal tDCS did not modulate GABA within the stimulated left M1 (main effect of stimulation condition: $F_{(1,6)}=1.410, p=$ 0.280 ; no significant main effect of time: $F_{(3,18)}=1.102, p=$ 0.374 ; no stimulation condition by time interaction: $F_{(3,18)}=0.517$, $p=0.676$ ), but there was a significant decrease in GABA within the nonstimulated right $\mathrm{M} 1$ (main effect of stimulation condition: $F_{(1,9)}=13.106, p=0.006$; no significant main effect of time: $F_{(3,27)}=$ 1.530, $p=0.229$; no stimulation condition by time interaction: $F_{(3,27)}=0.455, p=0.716$; Fig. $\left.2 B\right)$.

Bilateral tDCS did not modulate GABA within the anodetargeted left M1 (main effect of stimulation condition: $F_{(1,7)}=$ $0.894, p=0.376$; no significant main effect of time: $F_{(3,21)}=2.913$, $p=0.058$; no stimulation by time interaction: $F_{(3,21)}=0.062, p=$ 0.979 ), but there was a significant decrease in GABA within the cathode-targeted right M1 (main effect of stimulation condition: $F_{(1,9)}=8.058, p=0.019$; no significant effect of time: $F_{(3,27)}=$ $0.382, p=0.767$; no stimulation by time interaction: $F_{(3,27)}=$ 2.149, $p=0.117$; Fig. $2 C$ ).

\section{tDCS-related GABA changes in nonstimulated right M1 can} be explained by white matter microstructure.

Finally, we wished to investigate how change in GABA in the nonstimulated hemisphere during tDCS might relate to white matter microstructure between the two M1s. We first wished to investigate whether there was a correlation between tDCSinduced GABA change within the two M1s. No significant relationship was demonstrated for any of the stimulation conditions (anodal tDCS: $r_{(11)}=-0.014, p=0.965$; cathodal tDCS: $r_{(9)}=$ $-0.268, p=0.453$; bilateral: $\left.r_{(8)}=-0.117, p=0.765\right)$.

However, given the strong structural connectivity between the two M1s, we then went on to investigate whether tDCS-induced GABA change in the unstimulated M1 was related to the microstructure of white matter tracts connecting the two M1s on a subject-by-subject basis.

We demonstrated a significant relationship between the change in GABA during stimulation in the right (unstimulated) $\mathrm{M} 1$ and white matter microstructure between the stimulated and nonstimulated M1s, as assessed by FA for cathodal tDCS $\left(r_{(11)}=\right.$ $-0.610, p=0.035)$, but not for anodal tDCS $\left(r_{(11)}=0.487, p=\right.$ $0.108)$, or in the sham control $\left(r_{(11)}=-0.369, p=0.237\right)$ (Fig. $3 B)$. To assess the anatomical specificity of this result, we investigated the relationship between FA within the two control regions, the section of CC linking left M1 to right somatosensory cortex and the CST and change in GABA levels in right M1 in 
A

Anodal Stimulated Left M1

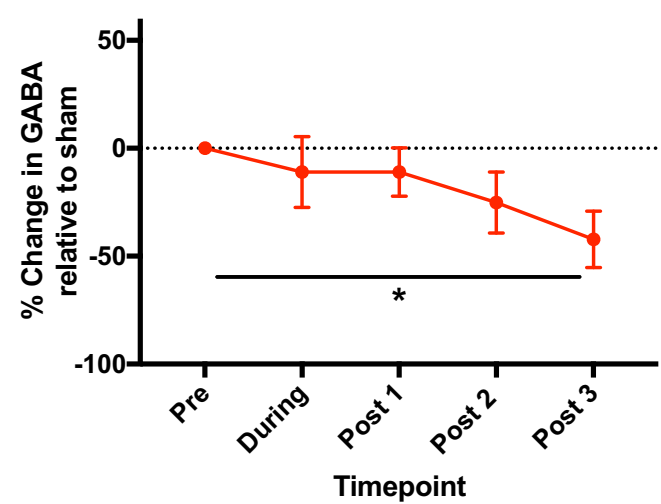

B

\section{Cathode Stimulated Left M1}

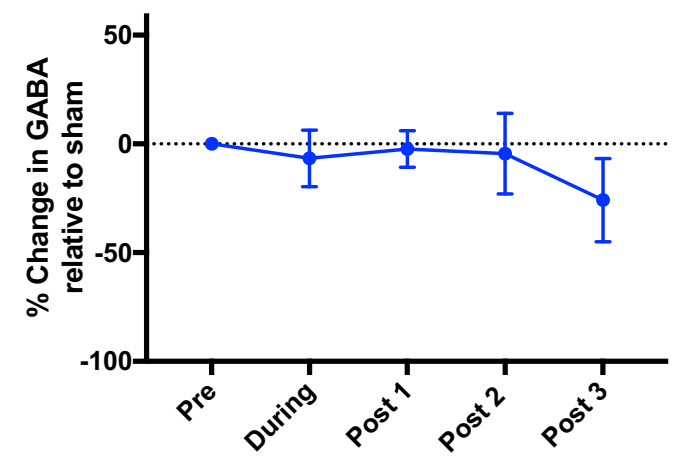

Timepoint

C

\section{Anode Targeted Left M1}

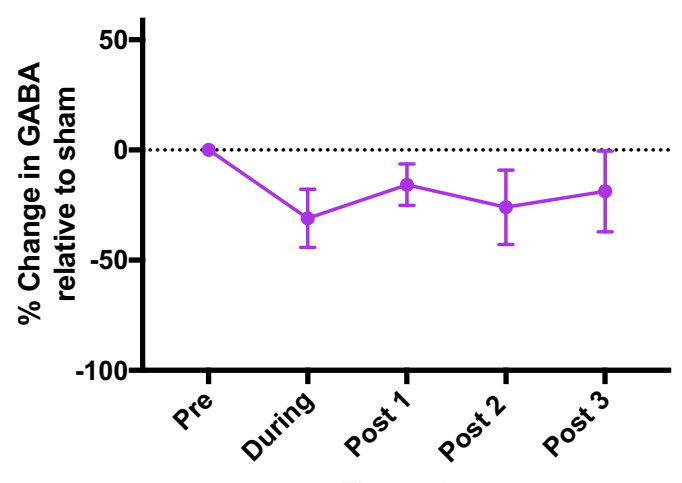

\section{Unstimulated Right M1}
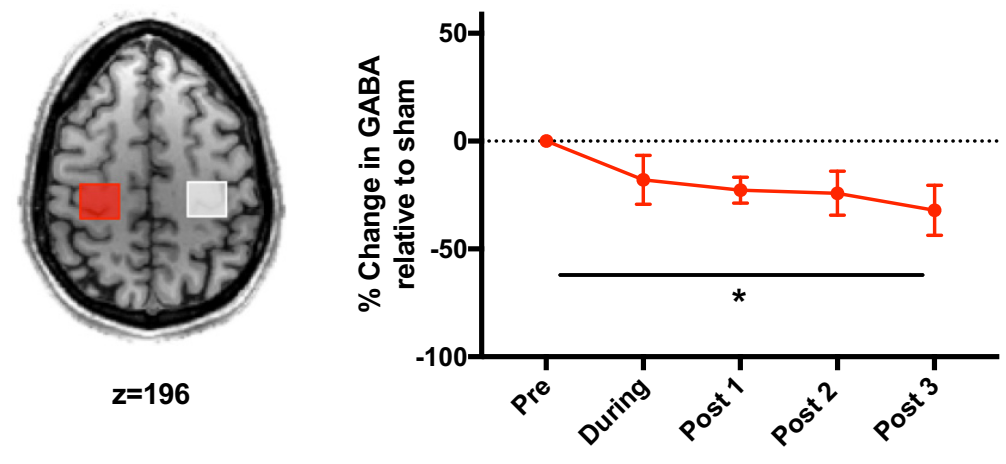

Timepoint
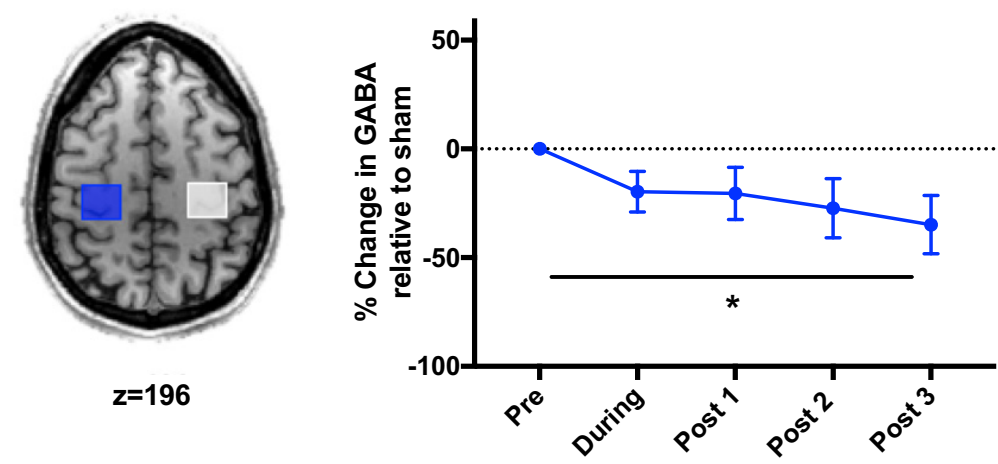

Timepoint

Cathode Targeted Right M1
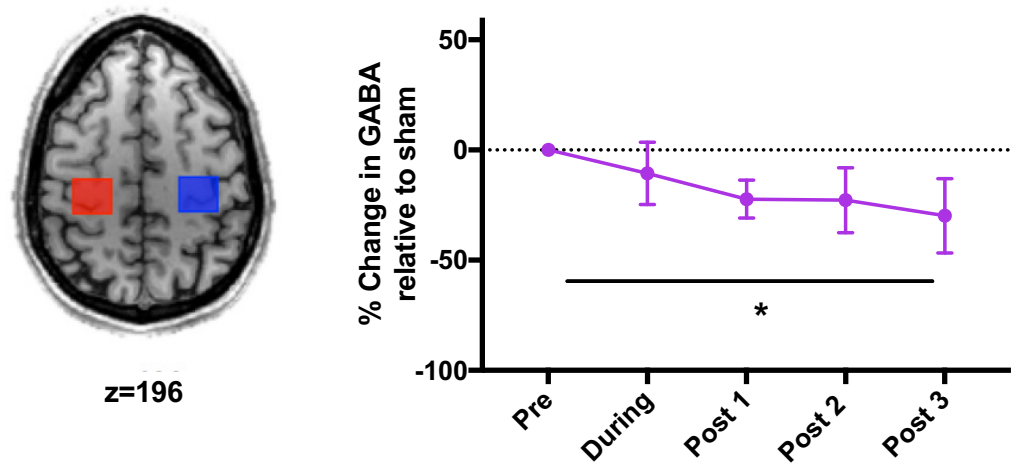

Timepoint

Figure 2. Percentage change in GABA during and after anodal $(\boldsymbol{A})$, cathodal $(\boldsymbol{B})$, and bilateral $(\boldsymbol{C})$ stimulation relative to sham stimulation. Brain images show the location of MRS voxels and stimulation. Red indicates anode placement, blue cathode placement, and gray no electrode placement. * Significant main effect of condition for each sham versus real stimulation comparison of the baselined GABA concentrations. Error bars indicate SEM. $A$, Decrease in GABA was observed in both stimulated and nonstimulated M1s in response to anodal tDCS (right M1, $n=12$; left M1, $n=$ 9). $\boldsymbol{B}$, Significant decrease in GABA was observed only within the nonstimulated right M1 in the cathodal tDCS condition (right M1, $n=10$; left M1, $n=7$ ). $\boldsymbol{C}$, Significant decrease in GABA was observed only within the cathode-targeted right M1 in response to bilateral tDCS condition (right M1, $n=10$; left M1, $n=8$ ). 
A
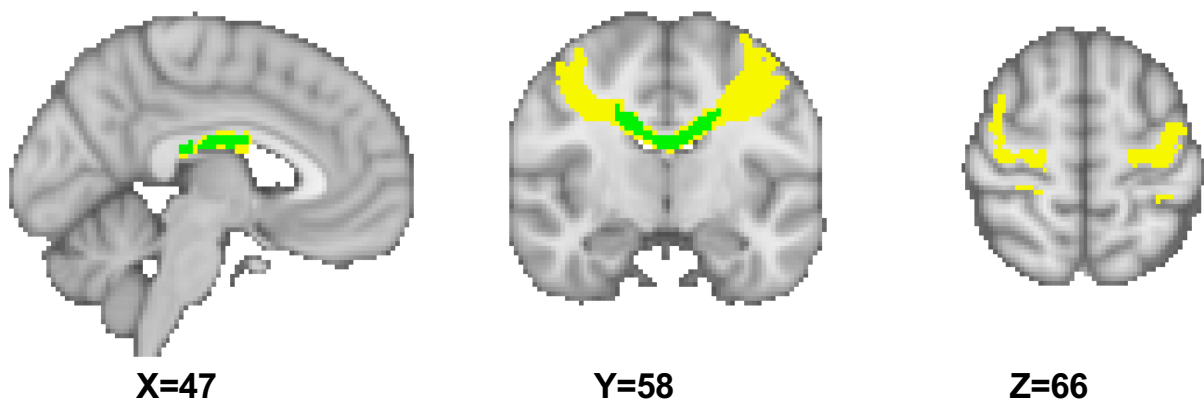

B

Anodal GABA change

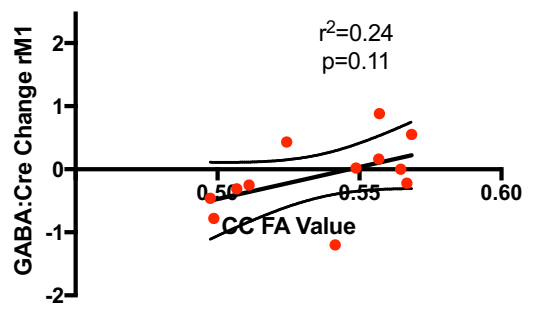

Cathodal GABA change

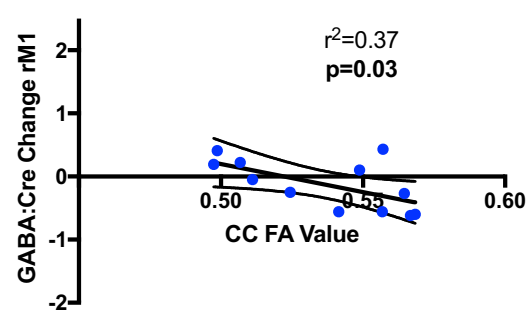

Sham GABA change

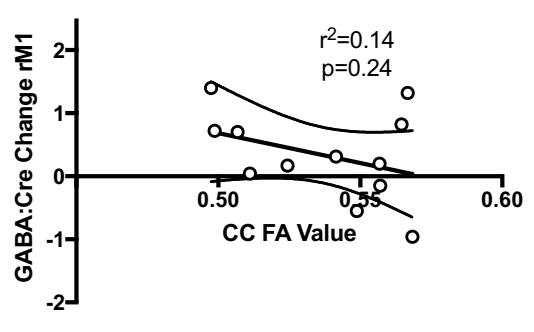

Figure 3. $\quad A, M 1-M 1 C C$ mask. The M1-M1 tract is shown in yellow and the M1-M1 CC in green. $B$, Cathodal $\mathrm{DDCS}$-induced GABA change within right $\mathrm{M} 1$ correlates with FA in the M1-M1 CC mask. No significant relationship between M1-M1 CCFA and change in GABA in right M1 was demonstrated during either anodal or cathodal tDCS.

response to stimulation, and demonstrated no significant correlations (CC: anodal: $r_{(11)}=0.435, p=0.158$; cathodal: $r_{(11)}=$ $-0.325, p=0.302$; sham: $r_{(11)}=-0.575, p=0.051$; CST: anodal: $r_{(11)}=0.160, p=0.620$; cathodal: $r_{(11)}=-0.069, p=0.831$; sham: $\left.r_{(11)}=-0.138, p=0.668\right)$. Furthermore, no significant correlation was found for any stimulation condition between changes in glutamate levels in right $\mathrm{M} 1$ and FA in either the M1-M1 CC mask (anodal: $r_{(11)}=0.382, p=0.221$; cathodal: $r_{(11)}=-0.472, p=0.121$; sham: $\left.r_{(11)}=0.056, p=0.862\right)$ or CST mask (anodal: $r_{(11)}=-0.096, p=0.767$; cathodal: $r_{(11)}=$ $-0.453, p=0.139$; sham: $\left.r_{(11)}=0.445, p=0.147\right)$.

\section{Discussion}

This study was performed to explore the physiological changes underpinning motor plasticity across the motor network. We chose to use tDCS as our plasticity-induction protocol as it enabled us to directly target one M1 while studying the neurochemical changes in both M1s. We have replicated previous findings (Stagg et al., 2009; Stagg and Nitsche, 2011; Kim et al., 2014; Bachtiar et al., 2015) that anodal tDCS applied to M1 causes a decrease in MRS-measured GABA levels in the stimulated area of cortex, but we have also shown for the first time that this decrease is accompanied by a concurrent decrease in GABA in the nonstimulated M1. Cathodal tDCS led to a significant decrease in GABA in the nonstimulated right $\mathrm{M} 1$, though with no significant GABA changes in the cathode-targeted left M1. Bilateral tDCS resulted in a GABA decrease only in the cathode-targeted right M1.

Our findings are in broad agreement with that of O'Shea et al. (2014), who used TMS to examine changes to cortical excitability in both M1s after tDCS, which demonstrated significant increases in corticospinal excitability in both hemispheres following anodal stimulation to left M1, consistent with our finding of a bilateral GABA decrease. The same study found a decrease in corticospinal excitability in the stimulated hemisphere in the cathodal condition, where we found no change in GABA, but an increase in excitability in nonstimulated hemisphere, which is consistent with our finding of a GABA decrease.

For all conditions in which a GABA decrease was observed, the change persisted into the final MRS measurement. This indicates that $\mathrm{tDCS}$-induced changes in neurochemicals endure for at least $30 \mathrm{~min}$ after stimulation. This is perhaps unsurprising given that effects of anodal tDCS on corticospinal excitability have been shown to outlast stimulation by up to $90 \mathrm{~min}$ (Nitsche and Paulus, 2001). Further studies over longer timescales are therefore required to determine the duration of tDCS-induced GABA changes.

We found no effect of stimulation on glutamate concentration in either hemisphere. A previous study has demonstrated changes in glutamate concentration in the stimulated region with cathodal tDCS to M1 (Stagg et al., 2009), but another failed to find the same effect (Kim et al., 2014). A recent review examined the influence of factors such as field strength, experimental design, and sequence on MRS-measured changes in glutamate (Mullins, 2018); it may be that differences in design and acquisition time of tDCS-MRS studies may be responsible for the differing results. Futhermore, as is common in many tDCS-MRS studies, we had a relatively small sample size. Replicating this paradigm with a larger sample size will be important to allow robust conclusions to be drawn.

Bilateral decrease in M1 GABA with anodal tDCS to left M1 Anodal tDCS has been shown to induce LTP-like plasticity in M1 in a manner at least similar to the mechanisms underpinning motor learning and recovery after stroke (Bindman et al., 1964; Weiss et al., 1998; Stagg and Nitsche, 2011; Di Lazzaro et al., 2012). Anodal tDCS therefore provided an experimental model 
with which to explore neurochemical changes across the wider motor network in response to a specific plasticity-induction protocol and has suggested a decrease in GABA as a common mediating factor across the motor network. Consistent with this hypothesis, decreases in GABA in M1 have been demonstrated when individuals practice a motor skill (Floyer-Lea et al., 2006) and tDCS-induced changes in MRS measured GABA within M1 have been shown to correlate with individuals' ability to learn motor tasks (Stagg et al., 2011a; Kim et al., 2014), whereas the same correlations were not seen with MRS-measured glutamate (Kim et al., 2014). These results highlight the potential importance of change in GABA concentration in mediating motor plasticity and examining GABA changes during learning under tDCS would be an important avenue to explore in future studies.

Here, we saw a decrease in GABA in both the anodal stimulated and nonstimulated hemispheres, suggesting for the first time that a decrease in GABA outside of the stimulated M1 may be important for motor plasticity. However, there was no significant relationship between the change in GABA in the nonstimulated hemisphere and the white matter microstructure between the two M1s in the anodal condition. Although this is perhaps a surprising result at first sight, it would be consistent with the hypothesis that the interhemispheric effects of anodal tDCS rely on facilitation between the two M1s. Because the majority of the transcallosal fibers from M1 to M1 project onto local inhibitory interneurons in the hand area (Schnitzler et al., 1996), it is therefore perhaps not surprising that the microstructure of this tract does not relate to GABA change that appears to be caused by interhemispheric facilitation, though this explanation is speculative and requires further testing.

\section{Magnitude of GABA decrease in unstimulated hemisphere during to cathodal tDCS relates to underlying white matter microstructure}

Cathodal tDCS to the contralesional hemisphere has been suggested as a potential adjunct therapy in stroke recovery (Fregni et al., 2005; Boggio et al., 2007; Kim et al., 2010; Stagg et al., 2012). It has been hypothesized that cathodal tDCS improves function via a transcallosally mediated increase in excitatory activity in the contralateral M1 (Fregni et al., 2005; Boggio et al., 2006; Kim et al., 2010; Stagg et al., 2012; O'Shea et al., 2014) because cathodal tDCS has previously been demonstrated to decrease transcallosal inhibition between the motor cortices (Lang et al., 2004; Bradnam et al., 2010; Tazoe et al., 2014).

Here, we provide evidence to support this hypothesis by demonstrating that cathodal tDCS results in a decrease in inhibition in the contralateral M1 and, further, that this effect is positively correlated with the white matter microstructure between the two cortices such that a higher FA value in the CC was linked to a greater decrease in GABA levels within the right, nonstimulated M1. There is a strong positive correlation between an individuals' transcallosal inhibition and the microstructure of the M1-M1 callosal tracts as measured by FA (Wahl et al., 2007; Fling et al., 2013) and the link between greater FA within the CC and increased effect of tDCS in the unstimulated hemisphere is broadly consistent with previous findings showing a link between FA values within transcallosal fibers and tDCS-induced fMRI laterality index during a motor task (Lindenberg et al., 2013). Together, these findings may provide an explanation for some of the variability in response to cathodal tDCS in the stroke recovery literature and more generally (Chew et al., 2015; López-Alonso et al., 2015) and raises a hypothesis to test: that stroke patients with relatively structurally intact callosal fibers (higher FA) may ben- efit from cathodal tDCS applied to the contralesional M1, whereas those with less structural integrity (lower FA) in their callosal fibers may respond better to other approaches.

For all participants in this study, the stimulation was applied to the dominant hemisphere, so it may not be possible to generalize these findings to individuals who are left-handed or to stimulation of the nondominant hemisphere.

\section{Physiological effects of bilateral tDCS are not simply a sum of those underpinning anodal and cathodal tDCS}

Bilateral tDCS led only to a decrease in GABA in the cathodetargeted M1. Although this finding is somewhat difficult to interpret, it is important to highlight that, consistent with previous studies (O'Shea et al., 2014; Waters et al., 2017), bilateral stimulation cannot simply be explained as the combined effect of the anode and cathode conditions. In the bilateral M1 montage, current flows transversely across the cortex, as opposed to the radial current flow direction in the unilateral montages. Recent work by Rawji et al. (2018) has shown that the orientation of current flow across the motor cortex influences corticospinal excitability, with current flowing orthogonal to the central sulcus eliciting the greatest changes. It has previously been proposed that this difference in current flow direction between the bilateral and unilateral montages may alter the influence of the tDCS polarity on behavioral (Waters et al., 2017) and neurophysiological (O'Shea et al., 2014) metrics, a hypothesis that would be supported by the data presented here. Together, these findings suggest that further data are needed before bilateral tDCS can be fully optimized as a neuroscientifically informed adjunct therapy in stroke recovery.

\section{Conclusions}

These results show for the first time that neurochemical changes are observed outside of the targeted primary motor cortex during plasticity induction in humans, supporting the role of the wider motor network in motor plasticity. The importance of the wider motor network in plasticity is further highlighted by the relationship demonstrated between the anatomically distant effects of cathodal tDCS and the underlying white matter microstructure, findings that, together, begin to explain the putative effectiveness and the well described variability of tDCS in stroke recovery.

\section{References}

Allman C, Amadi U, Winkler AM, Wilkins L, Filippini N, Kischka U, Stagg CJ, Johansen-Berg H (2016) Ipsilesional anodal tDCS enhances the functional benefits of rehabilitation in patients after stroke. Sci Transl Med 8:330re1. CrossRef Medline

Amadi U, Allman C, Johansen-Berg H, Stagg CJ (2015) The homeostatic interaction between anodal transcranial direct current stimulation and motor learning in humans is related to GABA A activity. Brain Stimul 8:898-905. CrossRef Medline

Andersson JL, Jenkinson M, Smith S (2007a) Non-linear registration, aka Spatial normalisation FMRIB technical report TR07JA2. FMRIB Anal Group Univ Oxf 2. Available at https://www.fmrib.ox.ac.uk/datasets/ techrep/tr07ja2/tr07ja2.pdf.

Andersson JL, Jenkinson M, Smith S (2007b) Non-linear optimisation. FMRIB technical report TR07JA1. Univ Oxf FMRIB Cent Oxf UK.

Bachtiar V, Near J, Johansen-Berg H, Stagg CJ (2015) Modulation of GABA and resting state functional connectivity by transcranial direct current stimulation. eLife 4:e08789. CrossRef Medline

Beckmann CF, DeLuca M, Devlin JT, Smith SM (2005) Investigations into resting-state connectivity using independent component analysis. Philos Trans R Soc B Biol Sci 360:1001-1013. CrossRef Medline

Behrens TE, Woolrich MW, Jenkinson M, Johansen-Berg H, Nunes RG, Clare S, Matthews PM, Brady JM, Smith SM (2003) Characterization and propagation of uncertainty in diffusion-weighted MR imaging. Magn Reson Med 50:1077-1088. CrossRef Medline 
Behrens TE, Berg HJ, Jbabdi S, Rushworth MF, Woolrich MW (2007) Probabilistic diffusion tractography with multiple fibre orientations: what can we gain? Neuroimage 34:144-155. CrossRef Medline

Bindman LJ, Lippold O, Redfearn J (1964) The action of brief polarizing currents on the cerebral cortex of the rat (1) during current flow and (2) in the production of long-lasting after-effects. J Physiol 172:369-382. CrossRef Medline

Boggio PS, Castro LO, Savagim EA, Braite R, Cruz VC, Rocha RR, Rigonatti SP, Silva MT, Fregni F (2006) Enhancement of non-dominant hand motor function by anodal transcranial direct current stimulation. Neurosci Lett 404:232-236. CrossRef Medline

Boggio PS, Nunes A, Rigonatti SP, Nitsche MA, Pascual-Leone A, Fregni F (2007) Repeated sessions of noninvasive brain DC stimulation is associated with motor function improvement in stroke patients. Restor Neurol Neurosci 25:123-129. Medline

Bradnam LV, Stinear CM, Lewis GN, Byblow WD (2010) Task-dependent modulation of inputs to proximal upper limb following transcranial direct current stimulation of primary motor cortex. J Neurophysiol 103: 2382-2389. CrossRef Medline

Cabanes E, Confort-Gouny S, Le Fur Y, Simond G, Cozzone PJ (2001) Optimization of residual water signal removal by HLSVD on simulated short echo time proton MR spectra of the human brain. J Magn Reson 150:116125. CrossRef Medline

Cabral J, Hugues E, Sporns O, Deco G (2011) Role of local network oscillations in resting-state functional connectivity. Neuroimage 57:130-139. CrossRef Medline

Chew T, Ho KA, Loo CK (2015) Inter-and intra-individual variability in response to transcranial direct current stimulation (tDCS) at varying current intensities. Brain Stimul 8:1130-1137. CrossRef Medline

Cuypers K, Leenus DJ, van den Berg FE, Nitsche MA, Thijs H, Wenderoth N, Meesen RL (2013) Is motor learning mediated by tDCS intensity? PLoS One 8:e67344. CrossRef Medline

Di Lazzaro V, Manganelli F, Dileone M, Notturno F, Esposito M, Capasso M, Dubbioso R, Pace M, Ranieri F, Minicuci G, Santoro L, Uncini A (2012) The effects of prolonged cathodal direct current stimulation on the excitatory and inhibitory circuits of the ipsilateral and contralateral motor cortex. J Neural Transm 119:1499-1506. CrossRef Medline

Di Pino G, Pellegrino G, Assenza G, Capone F, Ferreri F, Formica D, Ranieri F, Tombini M, Ziemann U, Rothwell JC, Di Lazzaro V (2014) Modulation of brain plasticity in stroke: a novel model for neurorehabilitation. Nat Rev Neurol 10:597-608. CrossRef Medline

Fling BW, Benson BL, Seidler RD (2013) Transcallosal sensorimotor fiber tract structure-function relationships. Hum Brain Mapp 34:384-395. CrossRef Medline

Floyer-Lea A, Wylezinska M, Kincses T, Matthews PM (2006) Rapid modulation of GABA concentration in human sensorimotor cortex during motor learning. J Neurophysiol 95:1639-1644. CrossRef Medline

Fregni F, Boggio PS, Mansur CG, Wagner T, Ferreira MJ, Lima MC, Rigonatti SP, Marcolin MA, Freedman SD, Nitsche MA, Pascual-Leone A (2005) Transcranial direct current stimulation of the unaffected hemisphere in stroke patients. Neuroreport 16:1551-1555. CrossRef Medline

Fritsch B, Reis J, Martinowich K, Schambra HM, Ji Y, Cohen LG, Lu B (2010) Direct current stimulation promotes BDNF-dependent synaptic plasticity: potential implications for motor learning. Neuron 66:198-204. CrossRef Medline

Galea JM, Vazquez A, Pasricha N, de Xivry JJ, Celnik P (2011) Dissociating the roles of the cerebellum and motor cortex during adaptive learning: the motor cortex retains what the cerebellum learns. Cereb Cortex 21:17611770. CrossRef Medline

Hall SD, Stanford IM, Yamawaki N, McAllister CJ, Rönnqvist KC, Woodhall GL, Furlong PL (2011) The role of GABAergic modulation in motor function related neuronal network activity. Neuroimage 56:1506-1510. CrossRef Medline

Hess G, Aizenman CD, Donoghue JP (1996) Conditions for the induction of long-term potentiation in layer II/III horizontal connections of the rat motor cortex. J Neurophysiol 75:1765-1778. CrossRef Medline

Hummel F, Cohen LG (2005) Improvement of motor function with noninvasive cortical stimulation in a patient with chronic stroke. Neurorehabil Neural Repair 19:14-19. CrossRef Medline

Hummel F, Celnik P, Giraux P, Floel A, Wu WH, Gerloff C, Cohen LG (2005) Effects of non-invasive cortical stimulation on skilled motor function in chronic stroke. Brain 128:490-499. CrossRef Medline
Jenkinson M, Bannister P, Brady M, Smith S (2002) Improved optimization for the robust and accurate linear registration and motion correction of brain images. Neuroimage 17:825-841. CrossRef Medline

Jenkinson M, Beckmann CF, Behrens TE, Woolrich MW, Smith SM (2012) FSL. Neuroimage 62:782-790. CrossRef Medline

Kim DY, Lim JY, Kang EK, You DS, Oh MK, Oh BM, Paik NJ (2010) Effect of transcranial direct current stimulation on motor recovery in patients with subacute stroke. Am J Phys Med Rehabil 89:879-886. CrossRef Medline

Kim S, Stephenson MC, Morris PG, Jackson SR (2014) tDCS-induced alterations in GABA concentration within primary motor cortex predict motor learning and motor memory: a $7 \mathrm{~T}$ magnetic resonance spectroscopy study. Neuroimage 99:237-243. CrossRef Medline

Kim YH, You SH, Ko MH, Park JW, Lee KH, Jang SH, Yoo WK, Hallett M (2006) Repetitive transcranial magnetic stimulation-induced corticomotor excitability and associated motor skill acquisition in chronic stroke. Stroke 37:1471-1476. CrossRef Medline

Lang N, Nitsche MA, Paulus W, Rothwell JC, Lemon RN (2004) Effects of transcranial direct current stimulation over the human motor cortex on corticospinal and transcallosal excitability. Exp Brain Res 156:439-443. CrossRef Medline

Lemke C, Hess A, Clare S, Bachtiar V, Stagg C, Jezzard P, Emir U (2015) Two-voxel spectroscopy with dynamic B0 shimming and flip angle adjustment at $7 \mathrm{~T}$ in the human motor cortex. NMR Biomed 28:852-860. CrossRef Medline

Lindenberg R, Renga V, Zhu LL, Nair D, Schlaug G (2010) Bihemispheric brain stimulation facilitates motor recovery in chronic stroke patients. Neurology 75:2176-2184. CrossRef Medline

Lindenberg R, Nachtigall L, Meinzer M, Sieg MM, Flöel A (2013) Differential effects of dual and unihemispheric motor cortex stimulation in older adults. J Neurosci 33:9176-9183. CrossRef Medline

López-Alonso V, Fernández-Del-Olmo M, Costantini A, Gonzalez-Henriquez JJ, Cheeran B (2015) Intra-individual variability in the response to anodal transcranial direct current stimulation. Clin Neurophysiol 126:2342-2347. CrossRef Medline

Monte-Silva K, Kuo MF, Hessenthaler S, Fresnoza S, Liebetanz D, Paulus W, Nitsche MA (2013) Induction of late LTP-like plasticity in the human motor cortex by repeated non-invasive brain stimulation. Brain Stimul 6:424-432. CrossRef Medline

Mullins PG (2018) Towards a theory of functional magnetic resonance spectroscopy (fMRS): a meta-analysis and discussion of using MRS to measure changes in neurotransmitters in real time. Scand J Psychol 59: 91-103. CrossRef Medline

Mullins PG, McGonigle DJ, O'Gorman RL, Puts NA, Vidyasagar R, Evans CJ, Edden RA (2014) Current practice in the use of MEGA-PRESS spectroscopy for the detection of GABA. Neuroimage 86:43-52. CrossRef Medline

Nitsche MA, Paulus W (2001) Sustained excitability elevations induced by transcranial DC motor cortex stimulation in humans. Neurology 57; 1899-1901. CrossRef Medline

Nitsche MA, Schauenburg A, Lang N, Liebetanz D, Exner C, Paulus W, Tergau F (2003) Facilitation of implicit motor learning by weak transcranial direct current stimulation of the primary motor cortex in the human. J Cogn Neurosci 15:619-626. CrossRef Medline

Nowak M, Hinson E, van Ede F, Pogosyan A, Guerra A, Quinn A, Brown P, Stagg CJ (2017) Driving human motor cortical oscillations leads to behaviourally relevant changes in local $\mathrm{GABA}_{\mathrm{A}}$ inhibition: a tACS-TMS study. J Neurosci 37:4481-4492. CrossRef Medline

Oldfield RC (1971) The assessment and analysis of handedness: the Edinburgh inventory. Neuropsychologia 9:97-113. CrossRef Medline

O'Shea J, Boudrias MH, Stagg CJ, Bachtiar V, Kischka U, Blicher JU, Johansen-Berg H (2014) Predicting behavioural response to TDCS in chronic motor stroke. Neuroimage 85:924-933. CrossRef Medline

Oz G, Tkáć I (2011) Short-echo, single-shot, full-intensity proton magnetic resonance spectroscopy for neurochemical profiling at $4 \mathrm{~T}$ : validation in the cerebellum and brainstem. Magn Reson Med 65:901-910. CrossRef Medline

Perez MA, Cohen LG (2009) Interhemispheric inhibition between primary motor cortices: what have we learned? J Physiol 587:725-726. CrossRef Medline

Provencher SW (2001) Automatic quantitation of localized in vivolH spectra with LCModel. NMR Biomed 14:260-264. CrossRef Medline

Rawji V, Ciocca M, Zacharia A, Soares D, Truong D, Bikson M, Rothwell J, 
Bestmann S (2018) tDCS changes in motor excitability are specific to orientation of current flow. Brain Stimul 11:289-298. CrossRef Medline

Reis J, Schambra HM, Cohen LG, Buch ER, Fritsch B, Zarahn E, Celnik PA, Krakauer JW (2009) Noninvasive cortical stimulation enhances motor skill acquisition over multiple days through an effect on consolidation. Proc Natl Acad Sci U S A 106:1590-1595. CrossRef Medline

Schnitzler A, Kessler KR, Benecke R (1996) Transcallosally mediated inhibition of interneurons within human primary motor cortex. Exp Brain Res 112:381-391. Medline

Smith SM (2002) Fast robust automated brain extraction. Hum Brain Mapp 17:143-155. CrossRef Medline

Smith SM, Jenkinson M, Woolrich MW, Beckmann CF, Behrens TE, Johansen-Berg H, Bannister PR, De Luca M, Drobnjak I, Flitney DE, Niazy RK, Saunders J, Vickers J, Zhang Y, De Stefano N, Brady JM, Matthews PM (2004) Advances in functional and structural MR image analysis and implementation as FSL. Neuroimage 23:S208-S219. CrossRef Medline

Soares DP, Law M (2009) Magnetic resonance spectroscopy of the brain: review of metabolites and clinical applications. Clin Radiol 64:12-21. CrossRef Medline

Sriraman A, Oishi T, Madhavan S (2014) Timing-dependent priming effects of tDCS on ankle motor skill learning. Brain Res 1581:23-29. CrossRef Medline

Stagg CJ, Nitsche MA (2011) Physiological basis of transcranial direct current stimulation. Neuroscientist 17:37-53. CrossRef Medline

Stagg CJ, Best JG, Stephenson MC, O'Shea J, Wylezinska M, Kincses ZT, Morris PG, Matthews PM, Johansen-Berg H (2009) Polarity-sensitive modulation of cortical neurotransmitters by transcranial stimulation. J Neurosci 29:5202-5206. CrossRef Medline

Stagg CJ, Bachtiar V, Johansen-Berg H (2011a) The role of GABA in human motor learning. Curr Biol 21:480-484. CrossRef Medline
Stagg CJ, Jayaram G, Pastor D, Kincses ZT, Matthews PM, Johansen-Berg H (2011b) Polarity and timing-dependent effects of transcranial direct current stimulation in explicit motor learning. Neuropsychologia 49:800804. CrossRef Medline

Stagg CJ, Bachtiar V, O’Shea J, Allman C, Bosnell RA, Kischka U, Matthews PM, Johansen-Berg H (2012) Cortical activation changes underlying stimulation-induced behavioural gains in chronic stroke. Brain 135:276284. CrossRef Medline

Tazoe T, Endoh T, Kitamura T, Ogata T (2014) Polarity specific effects of transcranial direct current stimulation on interhemispheric inhibition. PLoS One 9:e114244. CrossRef Medline

Tkác I, Starcuk Z, Choi IY, Gruetter R (1999) In vivo 1H NMR spectroscopy of rat brain at $1 \mathrm{~ms}$ echo time. Magn Reson Med 41:649-656. CrossRef Medline

Wahl M, Lauterbach-Soon B, Hattingen E, Jung P, Singer O, Volz S, Klein JC, Steinmetz H, Ziemann U (2007) Human motor corpus callosum: topography, Somatotopy, and Link between Microstructure and Function. J Neurosci 27:12132-12138. CrossRef Medline

Waters S, Wiestler T, Diedrichsen J (2017) Cooperation not competition: bihemispheric tDCS and fMRI show role for ipsilateral hemisphere in motor learning. J Neurosci 37:7500-7512. CrossRef Medline

Weiss SR, Eidsath A, Li XL, Heynen T, Post RM (1998) Quenching revisited: low level direct current inhibits amygdala-kindled seizures. Exp Neurol 154:185-192. CrossRef Medline

Woolrich MW, Jbabdi S, Patenaude B, Chappell M, Makni S, Behrens T, Beckmann C, Jenkinson M, Smith SM (2009) Bayesian analysis of neuroimaging data in FSL. Neuroimage 45:S173-S186. CrossRef Medline

Yousry TA, Schmid UD, Alkadhi H, Schmidt D, Peraud A, Buettner A, Winkler P (1997) Localization of the motor hand area to a knob on the precentral gyrus: a new landmark. Brain 120:141-157. CrossRef Medline 